\title{
Multiple radial solutions for Dirichlet problem involving two mean curvature equations in Euclidean and Minkowski spaces
}

Yaning Wang ${ }^{1 *}$

"Correspondence:

wyn051@163.com

'School of Mathematics and

Information Sciences, Henan

Normal University, Xinxiang,

P.R. China

\begin{abstract}
In this paper, we establish the existence of multiple positive radial solutions for the Dirichlet problem involving two mean curvature equations of spacelike graphs in Euclidean and Minkowski spaces.
\end{abstract}

MSC: 35J93; 35J25; 35A01; 53C42

Keywords: Dirichlet problem; Multiple radial solution; Mean curvature equation; Leggett-Williams' fixed point theorem

\section{Introduction}

A graph $\Sigma_{u}=\{(x, u(x)): x \in \Omega\}$ in the Euclidean space $\mathbb{R}^{N+1}$ is determined by a smooth function $u(x): \Omega \subseteq \mathbb{R}^{N} \rightarrow \mathbb{R}$. It is well known that the extrinsic mean curvature $H_{R}$ of $\Sigma_{u}$ is given by

$$
H_{R}=\frac{1}{N} \operatorname{div}\left(\frac{\nabla u}{\sqrt{1+|\nabla u|^{2}}}\right)
$$

where $\nabla$ and div are the gradient and the divergence operator in $\mathbb{R}^{N}$, respectively. The celebrated Bernstein's theorem (see [8]) says that the only entire graphs (i.e., $\Omega=\mathbb{R}^{2}$ ) in $\mathbb{R}^{3}$ with vanishing mean curvatures are necessarily the affine planes. Such a result holds for all $2 \leq N \leq 7$ but is no longer true for higher dimension (for more details, see [9]).

Let $\mathbb{L}^{N+1}=\left\{(t, x) \in \mathbb{R} \times \mathbb{R}^{N}\right\}$ be the flat Minkowski spacetime endowed with the Lorentzian metric $g_{L}=-d t^{2}+\sum_{i=1}^{N} d x_{i}^{2}$. A graph $\Sigma_{u}=\{(u(x), x): x \in \Omega\}$ in $\mathbb{L}^{N+1}$ is determined by a smooth function $u(x): \Omega \subseteq \mathbb{R}^{N} \rightarrow \mathbb{R}$. Usually, a graph in $\mathbb{L}^{N+1}$ is said to be spacelike if the norm of the gradient of $u$ is less than 1 , or equivalently, the induced metric on it from the ambient space $\mathbb{L}^{N+1}$ is Riemannian. The extrinsic mean curvature $H_{L}$ of a spacelike graph $\Sigma_{u}$ in $\mathbb{L}^{N+1}$ is given by

$$
H_{L}=\frac{1}{N} \operatorname{div}\left(\frac{\nabla u}{\sqrt{1-|\nabla u|^{2}}}\right)
$$

\section{Springer}


It has been proved by Calabi (see [10]) that the only entire spacelike graphs (i.e., $\Omega=\mathbb{R}^{N}$ ) in $\mathbb{L}^{N+1}, N \leq 4$, with vanishing mean curvatures are necessarily the hyperplanes. Later, Cheng and Yau [11] extended Calabi's theorem to arbitrary dimension.

In literature, (multiple) existence results for the Dirichlet problem involving prescribed mean curvature equations arising from (1.1) in $\mathbb{R}^{N+1}$ or (1.2) in $\mathbb{L}^{N+1}$ have been widely studied from various viewpoints and approaches. Among a large number of articles, we refer the reader to some recent surveys (see, for example, [4-6, 12-15, 20-22], and [27] related to (1.2) and [7, 17, 19, 23, 24], and [25] related to (1.1) and many references therein) for some related results on this topic.

Taking into account mean curvature equations (1.1) and (1.2), one can consider the following quasilinear partial differential equation:

$$
\operatorname{div}\left(\frac{\nabla u}{\sqrt{1+|\nabla u|^{2}}}\right)-\operatorname{div}\left(\frac{\nabla u}{\sqrt{1-|\nabla u|^{2}}}\right)=0
$$

which means that a spacelike graph has the same mean curvature both in $\mathbb{R}^{N+1}$ and $\mathbb{L}^{N+1}$. In [16], Kobayashi pointed out that the only spacelike surface satisfying $H_{R}=H_{L}=0$ for dimension two is an open piece of a spacelike plane or of a spacelike helicoid in the region. Recently, Albujer and Caballero [3] studied the $H_{R}=H_{L}$ surface equation and in particular they proved that the only spacelike graphs in $\mathbb{L}^{3}$, defined over a domain $\Omega \subseteq \mathbb{R}^{2}$ of infinite width, satisfying $H_{R}=H_{L}$ and asymptotic to a spacelike plane are (pieces of) spacelike planes. This result holds true for higher dimension, for more details, see [2]. Affine functions (whose gradient has norm less than 1) are clearly solutions to (1.3). Note that the existence of solutions to (1.3) with non-null mean curvature was proved in [1].

Inspired by the above results, in this paper we consider the Dirichlet problem of a new quasilinear elliptic partial differential equation

$$
\left\{\begin{array}{l}
\operatorname{div}\left(\frac{\nabla u}{\sqrt{1-|\nabla u|^{2}}}\right)-\operatorname{div}\left(\frac{\nabla u}{\sqrt{1+|\nabla u|^{2}}}\right)+f(|x|, u(x))=0 \quad \text { in } \Omega, \\
u(x)=0 \text { on } \partial \Omega
\end{array}\right.
$$

where $\Omega$ denotes the unit ball in $\mathbb{R}^{N}$ and $f:[0,1] \times[0,1) \rightarrow[0, \infty)$ is a nonnegative continuous function. From a geometric point of view, the function $f$ measures the proximity of the two different mean curvatures $H_{L}$ and $H_{R}$ of a spacelike graph in Minkowski and Euclidean spaces.

This paper is arranged as follows. In Section 2, we introduce the simplified LeggettWilliams's fixed point theorem. In Section 3, by applying this theorem, we establish several sufficient conditions for the Dirichlet problem (1.4) and the corresponding one-parameter problem (see Eq. (3.6)) to have multiple (at least three) positive radial solutions. Some corollaries are also given.

\section{Key lemmas}

In this section, we collect some necessary lemmas and notations. Let $E$ be a real Banach space and $P \subset E$ be a closed and convex subset. $P$ is said to be a (positive) cone if there hold

(i) $\lambda \geq 0$ and $x \in P \Rightarrow \lambda x \in P$;

(ii) $x \in P$ and $-x \in P \Rightarrow x=0$. 
Let $P \subset E$ be a cone. A map $\alpha$ is a nonnegative, continuous, and concave functional on $P$ if it satisfies the following two conditions:

(iii) $\alpha: P \rightarrow[0,+\infty)$ is continuous;

(iv) $\alpha(t x+(1-t) y) \geq t \alpha(x)+(1-t) \alpha(y)$ for any $x, y \in P$ and $0 \leq t \leq 1$.

For simplicity, we set $P_{c}:=\{x \in P:\|x\| \leq c\}$ and $P(\alpha, a, b)=\{x \in P: a \leq \alpha(x),\|x\| \leq b\}$. By a completely continuous map we mean a continuous function which takes bounded sets into relative compact sets. Now we are ready to give Leggett-Williams' fixed point theorem (see [18, Theorem 3.3]).

Lemma 2.1 ([18]) Let $P$ be a cone in a real Banach space $E$, and let $A: \bar{P}_{c} \rightarrow \bar{P}_{c}$ be completely continuous and $\alpha$ be a nonnegative continuous concave functional on $P$ with $\alpha(x) \leq\|x\|$ for any $x \in \bar{P}_{c}$. Suppose that there exist $0<a<b<d \leq c$ such that the following conditions hold:

(1) $\{x \in P(\alpha, b, d): \alpha(x)>b\} \neq \emptyset$ and $\alpha(A x)>b$ for all $x \in P(\alpha, b, d)$;

(2) $\|A x\|<$ a for any $x \in \bar{P}_{a}$;

(3) $\alpha(A x)>$ b for any $x \in P(\alpha, b, c)$ with $\|A x\|>d$.

Then $A$ has at least three fixed points $x_{1}, x_{2}$, and $x_{3}$ in $\bar{P}_{c}$ satisfying

$$
\left\|x_{1}\right\|<a, \quad b<\alpha\left(x_{2}\right), \quad\left\|x_{3}\right\|>a \quad \text { with } \alpha\left(x_{3}\right)<b .
$$

In particular, taking $c=d$, we obtain the simplified Leggett-Williams' fixed point theorem as follows.

Lemma 2.2 Let $P$ be a cone in a real Banach space $E$, and let $A: \bar{P}_{c} \rightarrow \bar{P}_{c}$ be completely continuous and $\alpha$ be a nonnegative continuous concave functional on $P$ with $\alpha(x) \leq\|x\|$ for any $x \in \bar{P}_{c}$. Suppose that there exist $0<a<b<c$ such that the following conditions hold:

(4) $\{x \in P(\alpha, b, c): \alpha(x)>b\} \neq \emptyset$ and $\alpha(A x)>b$ for all $x \in P(\alpha, b, c)$;

(5) $\|A x\|<$ a for any $x \in \bar{P}_{a}$.

Then $A$ has at least three fixed points $x_{1}, x_{2}$, and $x_{3}$ in $\bar{P}_{c}$ satisfying

$$
\left\|x_{1}\right\|<a, \quad b<\alpha\left(x_{2}\right), \quad\left\|x_{3}\right\|>a \quad \text { with } \alpha\left(x_{3}\right)<b .
$$

\section{Existence of multiple positive radial solutions}

Taking the radial coordinates $r=|x|$ and the change $u(x)=v(r)$, then (1.4) becomes the following boundary value problem:

$$
\left\{\begin{array}{l}
\left(r^{N-1} \phi\left(v^{\prime}\right)\right)^{\prime}=-r^{N-1} f(r, v(r)), \quad r \in(0,1), \\
v^{\prime}(0)=0, \quad v(1)=0,
\end{array}\right.
$$

where $N \geq 1$ and $\phi$ is defined by

$$
\phi(s)=\frac{s}{\sqrt{1-s^{2}}}-\frac{s}{\sqrt{1+s^{2}}}, \quad s \in(-1,1) .
$$

By a solution to (3.1) we mean a function $v \in C^{1}(0,1)$ with $\left\|v^{\prime}\right\|<1$ such that $r^{N-1} \phi\left(v^{\prime}\right)$ is differentiable and (3.1) is satisfied. Notice that existence results for solutions to (3.1) have 
been given in [6]. But the hypothesis on $f$ and techniques used in [6] are different from the ones used in this manuscript.

It is clear that $\phi(s)$ is odd and strictly increasing and $\phi(s) \in(0,+\infty)$ for all $s \in(0,1)$. Let us consider two arbitrary positive constants $t, s \in(0,1)$ such that $t s<1$. It is easily seen that

$$
\frac{s}{\sqrt{1-t^{2} s^{2}}}<\frac{s}{\sqrt{1-s^{2}}} \text { and } \frac{s}{\sqrt{1+t^{2} s^{2}}}>\frac{s}{\sqrt{1+s^{2}}}
$$

Thus, we have the following.

Lemma 3.1 For any positive constants $t, s \in(0,1)$ such that $t s<1$, we have

$$
t \phi(s)>\phi(t s)
$$

We consider the Banach space $E=C[0,1]$ with the supremum norm $\|\cdot\|$. Let $P=\{v(r) \in$ $E: v(r)$ is nonnegative, nonincreasing on $[0,1]$, and $\left.v^{\prime}(0)=v(1)=0\right\}$, then it is easy to check that $P$ is a cone in $E$. We define a nonlinear operator $A$ on $P \cap B_{1}$ as follows:

$$
(A v)(r)=\int_{r}^{1} \phi^{-1}\left(\frac{1}{t^{N-1}} \int_{0}^{t} s^{N-1} f(s, v(s)) d s\right) d t, \quad v(r) \in P \cap B_{1},
$$

where $B_{\rho}$ denotes the open ball in $E$, centered at 0 with radius $\rho>0$. By means of $\phi(-s)=$ $-\phi(s)$, we observe that $v$ is a positive radial solution of problem (3.1) if $v \in P \cap B_{1}$ is a fixed point of the nonlinear operator $A$. It is clear that $(A v)(r)$ is nonnegative for any $r \in[0,1]$. Also, we have

Lemma 3.2 $A\left(P \cap B_{1}\right) \subset P$.

Proof Since both $f$ and $\phi^{-1}$ are nonnegative, we get

$$
(A v)^{\prime}(r)=-\phi^{-1}\left(\frac{1}{r^{N-1}} \int_{0}^{r} s^{N-1} f(s, v(s)) d s\right) \leq 0
$$

for all $r \in(0,1]$. In view of $(A v)(1)=0$ and $(A v)^{\prime}(0)=0$, then $A v \in P$ for any $v \in P \cap B_{1}$.

The well-known Arzéla-Ascoli theorem says that if $M \subseteq C\left([0, T], \mathbb{R}^{n}\right)$ is uniformly bounded and equicontinuous, then $M$ is relatively compact in $C\left([0, T], \mathbb{R}^{n}\right)$. Following a standard argument in [6], we know that $A$ is compact on $P \cap \bar{B}_{\rho}$ for any $\rho \in(0,1)$. Since $f$ is continuous, following similar arguments in $[26,28]$, we have

Lemma 3.3 $A:\left(P \cap B_{1}\right) \rightarrow P$ is completely continuous.

Now we define a nonnegative continuous concave functional $\alpha: P \rightarrow[0,+\infty)$ by

$$
\alpha(v(r))=\min _{r \in[0, \eta]} v(r), \quad v(r) \in P
$$

where $\eta \in(0,1)$. Obviously, we have $\alpha(A v)=(A v)(\eta)$ for any $v \in P$.

Our main result is given as follows. 
Theorem 3.1 Assume that there exist four positive constants $a, b, c, \eta$ with $0<a<b<c<1$ and $b<1-\eta<1$ such that

$\left(\mathrm{C}_{1}\right) f(r, s) \leq N \phi(a)$ for all $(r, s) \in[0,1] \times[0, a]$;

$\left(C_{2}\right) f(r, s) \leq N \phi(c)$ for all $(r, s) \in[0,1] \times[0, c]$;

$\left(\mathrm{C}_{3}\right) f(r, s) \geq \frac{N}{\eta^{N}} \phi\left(\frac{b}{1-\eta}\right)$ for all $(r, s) \in[0, \eta] \times[b, c]$.

Then problem (3.1) has at least three positive radial solutions $u_{1}=v_{1}(r), u_{2}=v_{2}(r)$, and $u_{3}=v_{3}(r)$ such that

$$
\left\|v_{1}\right\|<a, \quad b<\alpha\left(v_{2}\right), \quad\left\|v_{3}\right\|>a \quad \text { with } \alpha\left(v_{3}\right)<b .
$$

Proof In what follows, we aim to prove that Lemma 2.2 is applicable under the above assumptions. Our discussion is divided into three steps.

Step 1. We show that $A \bar{P}_{c} \subset \bar{P}_{c}$. For any $v \in \bar{P}_{c}$, we have $v(r) \leq\|v\| \leq c$ for any $r \in[0,1]$, and hence by applying condition $\left(\mathrm{C}_{2}\right)$, we have

$$
\begin{aligned}
\|A v\| & =\int_{0}^{1} \phi^{-1}\left(\frac{1}{t^{N-1}} \int_{0}^{t} s^{N-1} f(s, v(s)) d s\right) d t \\
& \leq \int_{0}^{1} \phi^{-1}\left(\frac{1}{t^{N-1}} \int_{0}^{t} s^{N-1} N \phi(c) d s\right) d t \\
& =\int_{0}^{1} \phi^{-1}(\phi(c) t) d t \\
& <\int_{0}^{1} \phi^{-1}(\phi(c)) d t \\
& =c .
\end{aligned}
$$

This means that $A \bar{P}_{c} \subset \bar{P}_{c}$.

Step 2. We show that $\|A v\|<a$ for any $v \in \bar{P}_{a}$. Since the proof for this step is very similar to that of Step 1, with the aid of condition $\left(\mathrm{C}_{1}\right)$, we omit it.

Step 3. Let us consider $v:=\frac{b+c}{2}$. One can check easily that $v \in P,\|v\|\left\langle c\right.$, and $\left.\alpha(v)=\frac{b+c}{2}\right\rangle$ $b$. This implies that the set $\{v \in P(\alpha, b, c): \alpha(v)>b,\|v\|<c\}$ is nonempty. On the other hand, for any $v \in P(\alpha, b, c)$, we have $\alpha(v) \geq b,\|v\| \leq c$, and hence $b \leq \alpha(v) \leq v(r) \leq\|v\| \leq c$ for any $r \in[0, \eta]$. Thus, with the aid of condition $\left(\mathrm{C}_{3}\right)$, we have

$$
\begin{aligned}
\alpha(A v) & =\int_{\eta}^{1} \phi^{-1}\left(\frac{1}{t^{N-1}} \int_{0}^{t} s^{N-1} f(s, v(s)) d s\right) d t \\
& \geq \int_{\eta}^{1} \phi^{-1}\left(\frac{1}{t^{N-1}} \int_{0}^{\eta} s^{N-1} f(s, v(s)) d s\right) d t \\
& >\int_{\eta}^{1} \phi^{-1}\left(\frac{1}{t^{N-1}} \int_{0}^{\eta} s^{N-1} \frac{N}{\eta^{N}} \phi\left(\frac{b}{1-\eta}\right) d s\right) d t \\
& =\int_{\eta}^{1} \phi^{-1}\left(\frac{1}{t^{N-1}} \phi\left(\frac{b}{1-\eta}\right)\right) d t \\
& \geq \int_{\eta}^{1} \phi^{-1}\left(\phi\left(\frac{b}{1-\eta}\right)\right) d t \\
& =b .
\end{aligned}
$$


Taking into account Lemmas 3.2, 3.3, we see that now Lemma 2.2 is applicable and hence conclusions of Theorem 3.1 hold.

The application of Lemma 3.1 gives another existence result as follows.

Theorem 3.2 Assume that there exist four positive constants $a, b, c, \eta$ with $0<a<b<c<1$ and $2 b<1-\eta^{2}<1$ such that

$\left(\mathrm{C}_{1}\right) f(r, s) \leq N \phi(a)$ for all $(r, s) \in[0,1] \times[0, a]$;

$\left(\mathrm{C}_{2}\right) f(r, s) \leq N \phi(c)$ for all $(r, s) \in[0,1] \times[0, c]$;

$\left(\mathrm{C}_{4}\right) f(r, s) \geq N \phi\left(\frac{2 b}{1-\eta^{2}}\right)$ for all $(r, s) \in[0, \eta] \times[b, c]$.

Then problem (3.1) has at least three positive radial solutions $u_{1}, u_{2}$, and $u_{3}$ satisfying (3.4).

Proof Firstly, we show that if condition $\left(\mathrm{C}_{4}\right)$ is true, then condition (4) of Lemma 2.2 holds. Let $v \in P(\alpha, b, c)$; we have $\alpha(v) \geq b,\|v\| \leq c$ and hence $b \leq \alpha(v) \leq v(r) \leq\|v\| \leq c$ for any $r \in[0,1]$. Also, from the assumption we have $\frac{2 b}{1-\eta^{2}} t<1$ for any $t \in(\eta, 1)$. Thus, with the aid of condition $\left(\mathrm{C}_{4}\right)$, we have

$$
\begin{aligned}
\alpha(A v) & =\int_{\eta}^{1} \phi^{-1}\left(\frac{1}{t^{N-1}} \int_{0}^{t} s^{N-1} f(s, v(s)) d s\right) d t \\
& \geq \int_{\eta}^{1} \phi^{-1}\left(\frac{1}{t^{N-1}} \int_{0}^{t} s^{N-1} N \phi\left(\frac{2 b}{1-\eta^{2}}\right) d s\right) d t \\
& =\int_{\eta}^{1} \phi^{-1}\left(\phi\left(\frac{2 b}{1-\eta^{2}}\right) t\right) d t \\
& >\int_{\eta}^{1} \frac{2 b t}{1-\eta^{2}} d t \\
& =b,
\end{aligned}
$$

where in the last inequality we have used Lemma 3.1. The remaining proof follows directly from Theorem 3.1.

We continue considering some other conditions different from those in Theorems 3.1 and 3.2 as follows:

$\left(C_{5}\right) f^{1}:=\varlimsup_{s \rightarrow 1^{-}} \max _{r \in[0,1]} \frac{f(r, s)}{\phi(s)}<N$.

If the above condition holds, then there exist two positive constants $\theta, \delta$ with $0<f^{1}<\theta<N$ and $\delta>0$ such that $f(r, s) \leq \theta \phi(s)$ for all $(r, s) \in[0,1] \times(1-\delta, 1)$. We set $M:=\max \{f(r, s)$ : $(r, s) \in[0,1] \times[0,1-\delta]\}$. It follows immediately that

$$
f(r, s) \leq M+\theta \phi(s) \quad \text { for all }(r, s) \in[0,1] \times[0,1)
$$

Applying the above relation, together with Lemma 2.2, we have

Corollary 3.1 Assume that there exist four positive constants $a, b, c, \eta$ with $0<\phi^{-1}\left(\frac{M}{N-\theta}\right) \leq$ $a<b<c<1$ and $b<1-\eta<1$ such that conditions $\left(\mathrm{C}_{3}\right)$ and $\left(\mathrm{C}_{5}\right)$ hold. Then problem (3.1) has at least three positive radial solutions.

Proof Taking into account Theorem 3.1, we need only to prove that conditions $\left(\mathrm{C}_{1}\right)$ and $\left(\mathrm{C}_{2}\right)$ are true when assumption $\left(\mathrm{C}_{5}\right)$ holds. Because $0<\phi^{-1}\left(\frac{M}{N-\theta}\right) \leq a$ and both $\phi$ and $\phi^{-1}$ 
are strictly increasing, then we have $\frac{M}{N-\theta} \leq \phi(a)$ and hence $\phi^{-1}\left(\frac{M+\theta \phi(a)}{N}\right) \leq a$. For any $v \in \bar{P}_{a}$, we have $v(r) \leq\|v\| \leq a$ for any $r \in[0,1]$. Consequently, with the aid of (3.5), we obtain

$$
\begin{aligned}
\|A v\| & =\int_{0}^{1} \phi^{-1}\left(\frac{1}{t^{N-1}} \int_{0}^{t} s^{N-1} f(s, v(s)) d s\right) d t \\
& \leq \int_{0}^{1} \phi^{-1}\left(\frac{1}{t^{N-1}} \int_{0}^{t} s^{N-1}(M+\theta \phi(a)) d s\right) d t \\
& =\int_{0}^{1} \phi^{-1}\left(\frac{M+\theta \phi(a)}{N} t\right) d t \\
& <\int_{0}^{1} \phi^{-1}\left(\frac{M+\theta \phi(a)}{N}\right) d t \\
& \leq a .
\end{aligned}
$$

This implies that $A \bar{P}_{a} \subset \bar{P}_{a}$. Similarly, following the above calculation and the assumption $0<\phi^{-1}\left(\frac{M}{N-\theta}\right)<c<1$, we obtain $A \bar{P}_{c} \subset \bar{P}_{c}$. The remaining proof follows immediately from Theorem 3.1.

Next we consider a condition similar to that defined by $\left(\mathrm{C}_{5}\right)$ :

$\left(\mathrm{C}_{6}\right) f^{0}:=\varlimsup_{s \rightarrow 0^{+}} \max _{r \in[0,1]} \frac{f(r, s)}{\phi(s)}<N$.

If the above condition holds, then there exist two positive constants $\theta_{0}, \delta_{0}$ with $0<f^{0}<\theta_{0}<$ $N$ and $\delta_{0}>0$ such that $f(r, s) \leq \theta_{0} \phi(s)$ for all $(r, s) \in[0,1] \times\left(0, \delta_{0}\right)$. Let $M_{0}:=\max \{f(r, s)$ : $\left.(r, s) \in[0,1] \times\left[\delta_{0}, 1\right]\right\}$. It follows that

$$
f(r, s) \leq M_{0}+\theta_{0} \phi(s) \quad \text { for all }(r, s) \in[0,1] \times(0,1] .
$$

Applying (3.6), together with Lemma 2.2, we have the following.

Corollary 3.2 Assume that there exist four positive constants a, $b, c, \eta$ with $0<\phi^{-1}\left(\frac{M_{0}}{N-\theta_{0}}\right) \leq$ $a<b<c<1$ and $b<1-\eta<1$ such that conditions $\left(\mathrm{C}_{3}\right)$ and $\left(\mathrm{C}_{6}\right)$ hold. Then problem (3.1) has at least three positive radial solutions.

The proof for the above corollary is similar to that of Corollary 3.1, we omit it. From Theorem 3.1, we also establish the existence result of arbitrarily many positive radial solutions of problem (1.4).

Theorem 3.3 Assume that there exist some positive constants satisfying $0<a_{1}<b_{1}<c_{1} \leq$ $a_{2}<b_{2}<c_{2}<\cdots<c_{n-1} \leq a_{n}<1\left(n \in \mathbb{N}^{*}\right)$ and $b_{i}<1-\eta_{i}<1(1 \leq i \leq n)$ such that

$\left(\mathrm{C}_{7}\right) f(r, s) \leq N \phi\left(a_{i}\right)$ for all $(r, s) \in[0,1] \times\left[0, a_{i}\right]$;

$\left(\mathrm{C}_{8}\right) f(r, s) \leq N \phi\left(c_{i}\right)$ for all $(r, s) \in[0,1] \times\left[0, c_{i}\right]$

$\left(C_{9}\right) f(r, s) \geq \frac{N}{\eta_{i}^{N}} \phi\left(\frac{b_{i}}{1-\eta_{i}}\right)$ for all $(r, s) \in\left[0, \eta_{i}\right] \times\left[b_{i}, c_{i}\right]$.

Then problem (3.1) has at least $2 n-1$ positive radial solutions.

Proof When $n=1$, making use of condition $\left(C_{7}\right)$ and proof of Theorem 3.1, we have $A \bar{P}_{a_{1}} \subset \bar{P}_{a_{1}}$. By applying the Schauder fixed point theorem, problem (3.1) has at least one solution. When $n=2$, conclusions of Theorem 3.1 still hold if $c_{1}=a_{2}$. In this case, problem 
(3.1) has at least three solutions. Following such approaches, we conclude that problem (3.1) has at least $2 n-1$ positive radial solutions under the given assumptions.

According to Theorem 3.2, another version of the existence result of arbitrarily many positive radial solutions of problem (3.1) is given as follows.

Remark 3.1 If $b_{i}<1-\eta_{i}<1$ and $f(r, s) \geq \frac{N}{\eta_{i}^{N}} \phi\left(\frac{b_{i}}{1-\eta_{i}}\right)$ for all $(r, s) \in[0,1] \times\left[b_{i}, c_{i}\right]$ in Theorem 3.3 are replaced by $2 b_{i}<1-\eta_{i}^{2}<1$ and $f(r, s) \geq N \phi\left(\frac{2 b}{1-\eta_{i}^{2}}\right)$ for all $(r, s) \in\left[0, \eta_{i}\right] \times\left[b_{i}, c_{i}\right]$, respectively, then conclusion of Theorem 3.3 is still true provided that $\left(\mathrm{C}_{9}\right)$ is replaced by $f(r, s) \geq N \phi\left(\frac{2 b_{i}}{1-\eta_{i}^{2}}\right)$ for all $(r, s) \in[0,1] \times\left[b_{i}, c_{i}\right]$.

Before closing this paper, let us consider the Dirichlet problem of the corresponding one-parameter problem of (1.4) as the following:

$$
\left\{\begin{array}{l}
\operatorname{div}\left(\frac{\nabla u}{\sqrt{1+|\nabla u|^{2}}}\right)-\operatorname{div}\left(\frac{\nabla u}{\sqrt{1-|\nabla u|^{2}}}\right)+\lambda f(|x|, u(x))=0 \quad \text { in } \Omega \\
u(x)=0 \text { on } \partial \Omega
\end{array}\right.
$$

where $\Omega$ denotes the unit ball in $\mathbb{R}^{N}$ and $\lambda>0$ is a parameter. Taking the radial coordinates $r=|x|$ and the change $u(x)=v(r)$, then (3.7) becomes

$$
\left\{\begin{array}{l}
\left(r^{N-1} \phi\left(v^{\prime}\right)\right)^{\prime}=-\lambda r^{N-1} f(r, v(r)), \quad r \in(0,1) \\
v^{\prime}(0)=0, \quad v(1)=0
\end{array}\right.
$$

where $\phi$ is defined by (3.2).

Consider those constants $a, b, c, \epsilon, \eta$ defined in Theorem 3.1. For simplicity, we set

$$
f_{a}:=\frac{\phi(a)}{\max _{[0,1] \times[0, a]} f(r, s)}, \quad f_{c}:=\frac{\phi(c)}{\max _{[0,1] \times[0, c]} f(r, s)}, \quad f^{b}:=\frac{\phi\left(\frac{b}{1-\eta}\right)}{\min _{[0, \eta] \times[b, c]} f(r, s) \eta^{N}}
$$

From the proof of Theorem 3.1, we have the following.

Theorem 3.4 Suppose that there exist four positive constants $a, b, c, \eta$ satisfying $0<a<$ $b<c<1$ and $b<1-\eta<1$ such that $f^{b} \leq \min \left\{f_{a}, f_{c}\right\}$. If the parameter $\lambda$ satisfies

$$
f^{b}<\lambda<\min \left\{f_{a}, f_{c}\right\}
$$

then problem (3.7) has at least three positive radial solutions satisfying (3.4).

Consider those $a_{i}, b_{i}, c_{i}, \eta_{i}$ defined in Theorem 3.3, and let

$$
\begin{aligned}
f_{a, i} & :=\frac{\phi\left(a_{i}\right)}{\max _{[0,1] \times\left[0, a_{i}\right]} f(r, s)}, \quad f_{c_{i}, i}:=\frac{\phi\left(c_{i}\right)}{\max _{[0,1] \times\left[0, c_{i}\right]} f(r, s)}, \\
f^{b, i} & :=\frac{\phi\left(\frac{b_{i}}{1-\eta}\right)}{\min _{\left[0, \eta_{i}\right] \times\left[b_{i}, c_{i}\right]} f(r, s) \eta^{N}} .
\end{aligned}
$$

From Theorems 3.3, 3.4, we have the following. 
Corollary 3.3 Assume that there exist some positive constants satisfying $0<a_{1}<b_{1}<$ $c_{1} \leq a_{2}<b_{2}<c_{2}<\cdots<c_{n-1} \leq a_{n}<1\left(n \in \mathbb{N}^{*}\right)$ and $b_{i}<1-\eta_{i}<1(1 \leq i \leq n)$ such that $\max \left\{f^{b, 1}, \ldots, f^{b, n}\right\} \leq \min \left\{f_{a, 1}, \ldots, f_{a, n}, f_{c, 1}, \ldots, f_{c, n}\right\}$. If the parameter $\lambda$ satisfies

$$
\max \left\{f^{b, 1}, \ldots, f^{b, n}\right\}<\lambda<\min \left\{f_{a, 1}, \ldots, f_{a, n}, f_{c, 1}, \ldots, f_{c, n}\right\}
$$

then problem (3.7) has at least $2 n-1$ positive radial solutions.

By considering a special nonlinearity $f$, we construct an example to illustrate our main results.

Example 3.1 For any given $0<a<b<c<1$ and $\phi(s)=\frac{s}{\sqrt{1-s^{2}}}-\frac{s}{\sqrt{1+s^{2}}}$ with $s \in(0,1)$, since $\lim _{s \rightarrow 1^{-}} \phi(s)=+\infty$, then there exists positive $\eta$ (close enough to $1-b$ from left) such that $\phi(c)=\frac{1}{\eta^{N}} \phi\left(\frac{b}{1-\eta}\right)$. We denote by $\alpha=N \phi(c)$ and consider the following nonlinearity $f(r, s)$ defined by

$$
f(r, s)= \begin{cases}0, & s \in(0, a), \\ \frac{s-a}{b-a} \alpha, & s \in[a, b), \\ \alpha, & s \in[b, 1) .\end{cases}
$$

Therefore, applying Theorem 3.1, problem (3.1) with the above nonlinearity $f$ has at least three solutions.

\section{Acknowledgements}

The author would like to thank the handling editors and the anonymous reviewers for their useful suggestions.

\section{Funding}

Not applicable.

Availability of data and materials

Not applicable.

\section{Competing interests}

The author declares that he has no competing interests.

\section{Authors' contributions}

The author conceived of the study, drafted the manuscript, and approved the final manuscript.

\section{Publisher's Note}

Springer Nature remains neutral with regard to jurisdictional claims in published maps and institutional affiliations.

Received: 7 February 2019 Accepted: 22 April 2019 Published online: 30 April 2019

\section{References}

1. Alarcón, E.M., Albujer, A.L., Caballero, M.: On the solutions to the $H_{R}=H_{L}$ hypersurface equation. In: Proceedings Book of International Workshop on Theory of Submanifolds, Istanbul, Turkey, June 2-4, 2016, vol. 1 (2016)

2. Alarcón, E.M., Albujer, A.L., Caballero, M.: Spacelike hypersurfaces in the Lorentz-Minkowski space with the same Riemannian and Lorentzian mean curvature. In: Lorentzian Geometry and Related Topics. Springer Proceedings in Mathematics \& Statistics (2017)

3. Albujer, A.L., Caballero, M.: Geometric properties of surfaces with the same mean curvature in $\mathbb{R}^{3}$ and $\mathbb{L}^{3}$. J. Math. Anal. Appl. 445, 1013-1024 (2017)

4. Bereanu, C., Jebelean, P., Mawhin, J.: Radial solutions for some nonlinear problems involving mean curvature operators in Euclidean and Minkowski spaces. Proc. Am. Math. Soc. 137, 161-169 (2009)

5. Bereanu, C., Jebelean, P., Şerban, C.: Dirichlet problems with mean curvature operator in Minkowski space. In: New Trends in Differential Equations, Control Theory and Optimization: Proceedings of the 8th Congress of Romanian Mathematicians, pp. 1-20 (2016) 
6. Bereanu, C., Jebelean, P., Torres, P.J.: Positive radial solutions for Dirichlet problems with mean curvature operators in Minkowski space. J. Funct. Anal. 264, 270-287 (2013)

7. Bergner, M.: On the Dirichlet problem for the prescribed mean curvature equation over general domains. Differ. Geom. Appl. 27, 335-343 (2009)

8. Bernstein, S.: Sur un théorème de géométrie et ses applications aux équations aux dérivées partielles du type elliptique. Comm. Soc. Math. Kharkov 15, 38-45 (1915-1917)

9. Bombieri, E., Giorgi, E.D., Giusti, E.: Minimal cones and the Bernstein problem. Invent. Math. 7, 243-268 (1969)

10. Calabi, E.: Examples of Bernstein problems for some nonlinear equations. In: Global Analysis: Proc. Sympos. Pure Math., Berkeley, Calif., 1968, vol. XV, pp. 223-230. Am. Math. Soc., Providence (1970)

11. Cheng, S.Y., Yau, S.T.: Maximal space-like hypersurfaces in the Lorentz-Minkowski spaces. Ann. Math. (2) 104, 407-419 (1976)

12. Dai, G.: Bifurcation and positive solutions for problem with mean curvature operator in Minkowski space. Calc. Var. Partial Differ. Equ. 55, 1-17 (2016)

13. Dai, G.: Global bifurcation for problem with mean curvature operator on general domain. Nonlinear Differ. Equ. Appl. $24,1-10(2017)$

14. Dai, G.: Bifurcation and nonnegative solutions for problem with mean curvature operator on general domain. Indiana Univ. Math. J. 67, 2103-2121 (2018)

15. Dai, G., Wang, J.: Nodal solutions to problem with mean curvature operator in Minkowski space. Differ. Integral Equ. 30, 463-480 (2017)

16. Kobayashi, O.: Maximal surfaces in the 3-dimensional Minkowski space $\mathbb{L}^{3}$. Tokyo J. Math. 6, 297-309 (1983)

17. Le, V.K.: Some existence results on nontrivial solutions of the prescribed mean curvature equation. Adv. Nonlinear Stud. 5, 133-161 (2005)

18. Leggett, R.W., Williams, L.R.: Multiple positive fixed points of nonlinear operators on ordered Banach spaces. Indiana Univ. Math. J. 28, 673-688 (1979)

19. Li, Z., Ge, W.: New positive periodic solutions to singular Rayleigh prescribed mean curvature equations. Bound. Value Probl. 2017, Article ID 61 (2017)

20. Ma, R., Chen, T.: Multiple positive solutions for Dirichlet problem of prescribed mean curvature equations in Minkowski spaces. Electron. J. Differ. Equ. 2016, Article ID 180 (2016)

21. Ma, R., Gao, H., Lu, Y.: Global structure of radial positive solutions for a prescribed mean curvature problem in a ball. J. Funct. Anal. 270, 2430-2455 (2016)

22. Ma, R., Lu, Y.: Multiplicity of positive solutions for second order nonlinear Dirichlet problem with one-dimensional Minkowski-curvature operator. Adv. Nonlinear Stud. 15, 789-803 (2015)

23. Obersnel, F., Omari, P.: Existence and multiplicity results for the prescribed mean curvature equation via lower and upper solutions. Differ. Integral Equ. 22, 853-880 (2009)

24. Obersnel, F., Omari, P.: Positive solutions of the Dirichlet problem for the prescribed mean curvature equation. J. Differ. Equ. 249, 1674-1725 (2010)

25. Pan, H., Xing, R.: Bifurcation results for a class of prescribed mean curvature equations in bounded domains. Nonlinear Anal. 171, 21-31 (2018)

26. Papageorgiou, N.S., Radulescu, V.D., Repovs, D.D.: Nonlinear Analysis-Theory and Methods. Springer Monographs in Mathematics. Springer, Cham (2019)

27. Pei, M., Wang, L., LV, X.: Existence and multiplicity of positive solutions of a one-dimensional mean curvature equation in Minkowski space. Bound. Value Probl. 2018, Article ID 43 (2018)

28. Zhao, D., Wang, H., Ge, W.: Existence of triple positive solutions to a class of p-Laplacian boundary value problems. J. Math. Anal. Appl. 328, 972-983 (2007)

\section{Submit your manuscript to a SpringerOpen ${ }^{\circ}$ journal and benefit from:}

- Convenient online submission

- Rigorous peer review

- Open access: articles freely available online

- High visibility within the field

- Retaining the copyright to your article

Submit your next manuscript at $\gg$ springeropen.com 